\title{
Investigation of post-depositional processing of nitrate in East Antarctic snow: isotopic constraints on photolytic loss, re-oxidation, and source inputs
}

\author{
G. Shi et al.
}

Correspondence to: M. G. Hastings (meredith_hastings@ brown.edu) 
Supporting information includes two tables (Table S1 and Table S2), one figure (Fig. S1).

Table S1. Summary information for the seven snowpits presented in this study.

\begin{tabular}{|c|c|c|c|c|c|c|c|c|}
\hline Snowpit & Location & $\begin{array}{l}\text { Elevation, } \\
\mathrm{m}\end{array}$ & $\begin{array}{l}\text { Distance } \\
\text { from } \\
\text { coast, } \\
\mathrm{km}\end{array}$ & $\begin{array}{l}\text { Mean annual } \\
\text { accumulation, } \\
\mathrm{kg} \mathrm{m}^{-2} \mathrm{a}^{-11)}\end{array}$ & $\begin{array}{l}\text { Mean annual } \\
\text { Temperature, } \\
{ }^{\circ} \mathrm{C}^{2)}\end{array}$ & $\begin{array}{l}\text { Depth, } \\
\mathrm{cm}\end{array}$ & $\begin{array}{l}\text { Sampling } \\
\text { resolution, } \\
\mathrm{cm}\end{array}$ & $\begin{array}{l}\text { Sampling } \\
\text { date, } \\
\text { YYMM }\end{array}$ \\
\hline $\mathrm{P} 1$ & $\begin{array}{l}71.13^{\circ} \mathrm{S} \\
77.31^{\circ} \mathrm{E}\end{array}$ & 2037 & 200 & 172.0 & -29.12 & 150 & 3.0 & 201212 \\
\hline $\mathrm{P} 2$ & $\begin{array}{l}71.81^{\circ} \mathrm{S} \\
77.89^{\circ} \mathrm{E}\end{array}$ & 2295 & 283 & 99.4 & -32.87 & 200 & 5.0 & 201212 \\
\hline P3 & $\begin{array}{l}73.40^{\circ} \mathrm{S} \\
77.00^{\circ} \mathrm{E}\end{array}$ & 2545 & 462 & 90.7 & -35.72 & 200 & 5.0 & 201212 \\
\hline P4 & $\begin{array}{l}76.29^{\circ} \mathrm{S} \\
77.03^{\circ} \mathrm{E}\end{array}$ & 2843 & 787 & 54.8 & -41.28 & 200 & 2.0 & 201212 \\
\hline P5 & $\begin{array}{l}77.91^{\circ} \mathrm{S} \\
77.13^{\circ} \mathrm{E}\end{array}$ & 3154 & 968 & 33.3 & -46.37 & 200 & 2.0 & 201212 \\
\hline P6 & $\begin{array}{l}79.02^{\circ} \mathrm{S} \\
76.98 \mathrm{E}\end{array}$ & 3738 & 1092 & 25.4 & -53.13 & 200 & 2.5 & 201301 \\
\hline P7 & $\begin{array}{l}80.42^{\circ} \mathrm{S} \\
77.12^{\circ} \mathrm{E}\end{array}$ & 4093 & 1256 & 23.5 & -58.50 & 300 & 2.5 & 201301 \\
\hline
\end{tabular}

1) Mean annual snow accumulation rates are obtained from bamboo stick field measurements, updated to 2013 from Ding et al. (2011).

2) Mean annual temperatures are derived from $10 \mathrm{~m}$ borehole temperatures and automatic weather station observations (Ding et al., 2010). 
Table S2. Evaporative enrichment factors from the field experiment observations by Erbland et al. (2013) and modeled here following the theoretical approach of Frey et al. (2009). No ${ }^{18} \varepsilon$ data were reported by Erbland et al. (2013).

\begin{tabular}{|c|c|c|c|c|c|}
\hline \multirow{2}{*}{$\begin{array}{l}\text { Temperature, } \\
{ }^{\circ} \mathrm{C}\end{array}$} & \multicolumn{2}{|c|}{ Field Experiment } & \multirow{2}{*}{$\begin{array}{l}\text { Temperature, } \\
{ }^{\circ} \mathrm{C}\end{array}$} & \multicolumn{2}{|c|}{ Model } \\
\hline & ${ }^{15} \varepsilon, \%$ & ${ }^{18} \varepsilon, \%$ & & ${ }^{15} \varepsilon, \%$ & ${ }^{18} \varepsilon, \%$ \\
\hline-10 & $-3.6 \pm 1.1$ & - & 0 & 12.6 & 1.1 \\
\hline-20 & $-0.3 \pm 1.2$ & - & -13 & 13.2 & 1.0 \\
\hline \multirow[t]{3}{*}{-30} & $0.9 \pm 3.5$ & - & -33 & 14.2 & 0.8 \\
\hline & & & -53 & 15.3 & 0.7 \\
\hline & & & -73 & 16.8 & 0.6 \\
\hline
\end{tabular}




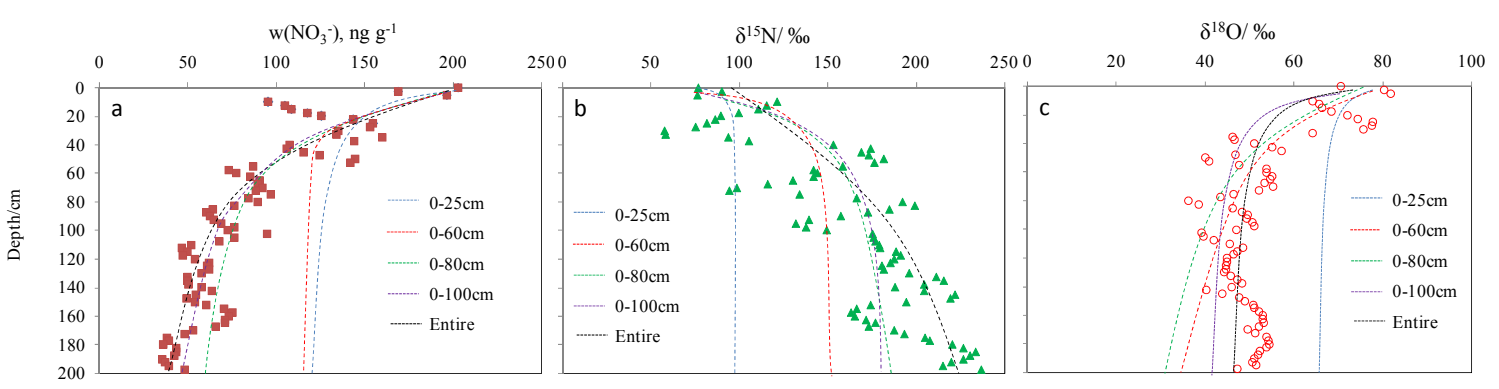

Fig. S1. Detailed profiles of $\mathrm{w}\left(\mathrm{NO}_{3}{ }^{-}\right), \delta^{15} \mathrm{~N}$ and $\delta^{18} \mathrm{O}$ in different snow depth intervals $(0-25 \mathrm{~cm}, 0-60 \mathrm{~cm}$, $0-80 \mathrm{~cm}, 0-100 \mathrm{~cm}$ and 0 -bottom) for snowpit P6. The dashed lines are the best fit regressions for the observed data, and asymptotic values are calculated for $\mathrm{w}\left(\mathrm{NO}_{3}{ }^{-}\right), \delta^{15} \mathrm{~N}$ and $\delta^{18} \mathrm{O}$ by Eq. (6). 
Snowpit P1

\begin{tabular}{|c|c|c|c|}
\hline Depth, cm & $\mathrm{w}\left(\mathrm{NO}_{3}{ }^{-}\right), \mathrm{ng} \mathrm{g}^{-1}$ & $\delta^{15} \mathrm{~N}, \% 0$ & $\delta^{18} \mathrm{O}, \%$ \\
\hline 150.0 & 57.7 & 13.0 & 77.4 \\
\hline 147.0 & 78.7 & 37.4 & 73.9 \\
\hline 144.0 & 79.7 & 59.4 & 69.2 \\
\hline 141.0 & 52.4 & 50.3 & 71.0 \\
\hline 138.0 & 36.2 & -5.8 & 78.2 \\
\hline 135.0 & 42.4 & 0.2 & 75.0 \\
\hline 132.0 & 41.5 & 13.9 & 71.6 \\
\hline 129.0 & 43.4 & 24.8 & 85.2 \\
\hline 126.0 & 44.8 & 24.1 & 89.5 \\
\hline 123.0 & 60.1 & 23.6 & 82.3 \\
\hline 120.0 & 74.9 & 19.1 & 78.0 \\
\hline 117.0 & 84.4 & 12.2 & 75.4 \\
\hline 114.0 & 81.6 & 10.1 & 73.4 \\
\hline 111.0 & 81.6 & 11.6 & 73.4 \\
\hline 108.0 & 72.5 & 15.6 & 77.8 \\
\hline 105.0 & 59.6 & 23.9 & 85.3 \\
\hline 102.0 & 46.2 & 23.6 & 85.4 \\
\hline 99.0 & 38.1 & 25.6 & 85.1 \\
\hline 96.0 & 33.8 & 33.7 & 83.8 \\
\hline 93.0 & 31.9 & 38.9 & 83.0 \\
\hline 90.0 & 31.4 & 43.1 & 78.5 \\
\hline 87.0 & 34.8 & 46.2 & 78.4 \\
\hline 84.0 & 30.0 & 47.3 & 75.6 \\
\hline 81.0 & 28.1 & 47.7 & 73.8 \\
\hline 78.0 & 26.7 & 50.6 & 74.1 \\
\hline 75.0 & 27.1 & 48.8 & 71.1 \\
\hline 72.0 & 28.1 & 45.5 & 70.4 \\
\hline 69.0 & 30.0 & 41.7 & 70.9 \\
\hline 66.0 & 33.8 & 10.0 & 69.5 \\
\hline 63.0 & 37.9 & 7.4 & 68.1 \\
\hline 60.0 & 42.9 & 4.8 & 66.7 \\
\hline 57.0 & 46.2 & 11.2 & 82.8 \\
\hline 54.0 & 50.1 & -3.8 & 78.9 \\
\hline 51.0 & 45.3 & -8.4 & 77.9 \\
\hline 48.0 & 36.7 & -14.8 & 76.9 \\
\hline 45.0 & 45.3 & -10.5 & 77.0 \\
\hline 42.0 & 43.9 & 11.0 & 96.3 \\
\hline 39.0 & 51.0 & 23.4 & 102.4 \\
\hline 36.0 & 47.7 & 26.3 & 102.0 \\
\hline 33.0 & 46.5 & 28.3 & 101.7 \\
\hline 30.0 & 44.8 & 30.1 & 105.3 \\
\hline
\end{tabular}




\begin{tabular}{|r|r|r|r|}
\hline 27.0 & 43.4 & 31.5 & 104.3 \\
\hline 24.0 & 40.0 & 32.4 & 101.6 \\
\hline 21.0 & 37.6 & 32.5 & 100.0 \\
\hline 18.0 & 35.7 & 33.8 & 98.9 \\
\hline 15.0 & 31.7 & 30.2 & 95.0 \\
\hline 12.0 & 27.6 & 27.1 & 91.7 \\
\hline 9.0 & 28.1 & 28.8 & 90.8 \\
\hline 6.0 & 37.2 & 27.0 & 85.3 \\
\hline 3.0 & 97.1 & 31.8 & 72.8 \\
\hline
\end{tabular}


Snowpit P2

\begin{tabular}{|c|c|c|c|}
\hline Depth, cm & $\mathrm{w}\left(\mathrm{NO}_{3}^{-}\right), \mathrm{ng} \mathrm{g}^{-1}$ & $\delta^{15} \mathrm{~N}, \%$ & $\delta^{18} \mathrm{O}, \%$ \\
\hline 5.0 & 74.5 & -5.7 & 91.7 \\
\hline 10.0 & 79.2 & -3.7 & 92.2 \\
\hline 15.0 & 54.1 & 25.5 & 90.0 \\
\hline 20.0 & 47.6 & 20.9 & 90.3 \\
\hline 25.0 & 52.1 & -1.9 & 89.8 \\
\hline 30.0 & 98.1 & 14.1 & 88.1 \\
\hline 35.0 & 173.0 & 17.8 & 86.7 \\
\hline 40.0 & 136.4 & 12.4 & 87.0 \\
\hline 45.0 & 99.4 & 1.2 & 75.7 \\
\hline 50.0 & 66.9 & 10.8 & 78.7 \\
\hline 55.0 & 81.9 & 53.4 & 92.5 \\
\hline 60.0 & 91.2 & 47.7 & 96.8 \\
\hline 65.0 & 90.7 & 35.5 & 95.5 \\
\hline 70.0 & 82.5 & 20.7 & 86.8 \\
\hline 75.0 & 108.4 & 30.3 & 76.3 \\
\hline 80.0 & 112.5 & 37.3 & 76.4 \\
\hline 85.0 & 99.4 & 35.4 & 78.9 \\
\hline 90.0 & 81.3 & 17.7 & 81.1 \\
\hline 95.0 & 85.8 & 21.5 & 83.9 \\
\hline 100.0 & 46.3 & -12.1 & 74.0 \\
\hline 105.0 & 49.8 & -11.6 & 73.6 \\
\hline 110.0 & 75.1 & 28.8 & 69.9 \\
\hline 115.0 & 72.6 & 24.0 & 71.3 \\
\hline 120.0 & 53.7 & 16.8 & 72.7 \\
\hline 125.0 & 46.3 & 12.7 & 72.7 \\
\hline 130.0 & 54.1 & 14.0 & 73.6 \\
\hline 135.0 & 69.4 & 23.6 & 75.5 \\
\hline 140.0 & 73.9 & 15.1 & 77.0 \\
\hline 145.0 & 92.0 & 9.5 & 76.7 \\
\hline 150.0 & 89.5 & 28.0 & 75.8 \\
\hline 155.0 & 69.4 & 53.4 & 85.1 \\
\hline 160.0 & 86.6 & 70.8 & 89.4 \\
\hline 165.0 & 74.7 & 34.0 & 83.4 \\
\hline 170.0 & 83.3 & 25.7 & 83.0 \\
\hline 175.0 & 88.7 & 25.7 & 81.8 \\
\hline 180.0 & 75.9 & 21.4 & 74.7 \\
\hline 185.0 & 38.5 & 34.2 & 70.4 \\
\hline 190.0 & 67.7 & 44.1 & 72.4 \\
\hline 195.0 & 66.9 & 40.5 & 74.4 \\
\hline 200.0 & 73.1 & 32.1 & 76.5 \\
\hline
\end{tabular}


Snowpit P3

\begin{tabular}{|c|c|c|c|}
\hline Depth, cm & $\mathrm{w}\left(\mathrm{NO}_{3}^{-}\right), \mathrm{ng} \mathrm{g}^{-1}$ & $\delta^{15} \mathrm{~N}, \%$ & $\delta^{18} \mathrm{O}, \%$ \\
\hline 5.0 & 266.3 & -2.2 & 79.0 \\
\hline 10.0 & 95.7 & 31.6 & 97.7 \\
\hline 15.0 & 105.5 & 39.9 & 104.0 \\
\hline 20.0 & 102.3 & 34.9 & 107.0 \\
\hline 25.0 & 117.1 & 23.4 & 76.8 \\
\hline 30.0 & 108.4 & 24.7 & 110.2 \\
\hline 35.0 & 86.6 & 9.8 & 97.1 \\
\hline 40.0 & 149.1 & 15.9 & 95.8 \\
\hline 45.0 & 95.3 & 35.6 & 85.2 \\
\hline 50.0 & 95.3 & 37.6 & 90.0 \\
\hline 55.0 & 109.0 & 27.6 & 95.0 \\
\hline 60.0 & 112.1 & 20.3 & 94.7 \\
\hline 65.0 & 112.9 & 16.1 & 100.9 \\
\hline 70.0 & 115.8 & 16.1 & 104.3 \\
\hline 75.0 & 101.4 & 25.4 & 107.9 \\
\hline 80.0 & 87.0 & 24.0 & 111.2 \\
\hline 85.0 & 59.5 & 11.3 & 104.7 \\
\hline 90.0 & 62.8 & 10.3 & 105.6 \\
\hline 95.0 & 92.4 & 23.6 & 89.2 \\
\hline 100.0 & 107.6 & 23.1 & 64.1 \\
\hline 105.0 & 79.4 & 24.6 & 83.0 \\
\hline 110.0 & 63.6 & 26.0 & 84.1 \\
\hline 115.0 & 65.2 & 24.4 & 81.0 \\
\hline 120.0 & 81.3 & 22.0 & 94.7 \\
\hline 125.0 & 71.8 & 20.2 & 87.7 \\
\hline 130.0 & 63.2 & 15.0 & 52.5 \\
\hline 135.0 & 68.1 & 17.2 & 81.5 \\
\hline 140.0 & 80.9 & 12.6 & 83.9 \\
\hline 145.0 & 82.9 & 11.8 & 75.9 \\
\hline 150.0 & 76.3 & 16.5 & 72.4 \\
\hline 155.0 & 86.8 & 27.7 & 70.8 \\
\hline 160.0 & 97.7 & 33.0 & 66.6 \\
\hline 165.0 & 64.0 & 22.4 & 70.7 \\
\hline 170.0 & 65.0 & 3.4 & 70.1 \\
\hline 175.0 & 66.1 & 15.0 & 57.8 \\
\hline 180.0 & 76.3 & 20.8 & 65.9 \\
\hline 185.0 & 75.1 & 20.3 & 58.3 \\
\hline 190.0 & 64.0 & 25.7 & 69.3 \\
\hline 195.0 & 61.1 & 24.9 & 70.6 \\
\hline 200.0 & & & \\
\hline
\end{tabular}


Snowpit P4

\begin{tabular}{|c|c|c|c|}
\hline Depth, cm & $\mathrm{w}\left(\mathrm{NO}_{3}^{-}\right), \mathrm{ng} \mathrm{g}^{-1}$ & $\delta^{15} \mathrm{~N}, \%$ & $\delta^{18} \mathrm{O}, \%$ \\
\hline 200.0 & 40.3 & 171.3 & 74.8 \\
\hline 198.0 & 38.0 & 174.1 & 75.1 \\
\hline 196.0 & 33.2 & 172.5 & 73.7 \\
\hline 194.0 & 27.4 & 168.2 & 70.4 \\
\hline 192.0 & 25.0 & 169.8 & 67.0 \\
\hline 190.0 & 25.4 & 175.8 & 65.0 \\
\hline 188.0 & 26.2 & 178.8 & 62.6 \\
\hline 186.0 & 25.4 & 176.7 & 61.8 \\
\hline 184.0 & 24.1 & 174.2 & 63.1 \\
\hline 182.0 & 26.6 & 169.8 & 62.1 \\
\hline 180.0 & 28.7 & 167.2 & 62.9 \\
\hline 178.0 & 28.9 & 163.0 & 62.9 \\
\hline 176.0 & 27.4 & 159.5 & 62.7 \\
\hline 174.0 & 34.1 & 148.8 & 64.1 \\
\hline 172.0 & 44.0 & 136.2 & 66.6 \\
\hline 170.0 & 42.7 & 125.9 & 67.2 \\
\hline 168.0 & 53.5 & 118.3 & 65.3 \\
\hline 166.0 & 63.0 & 113.1 & 64.8 \\
\hline 164.0 & 63.4 & 110.0 & 64.8 \\
\hline 162.0 & 65.0 & 109.8 & 65.0 \\
\hline 160.0 & 61.3 & 115.5 & 65.1 \\
\hline 158.0 & 58.0 & 125.6 & 64.5 \\
\hline 156.0 & 58.0 & 139.3 & 63.6 \\
\hline 154.0 & 58.4 & 150.2 & 62.9 \\
\hline 152.0 & 58.4 & 149.5 & 62.3 \\
\hline 150.0 & 68.8 & 139.5 & 63.9 \\
\hline 148.0 & 72.9 & 133.6 & 63.9 \\
\hline 146.0 & 65.9 & 123.3 & 64.5 \\
\hline 144.0 & 62.2 & 104.0 & 66.5 \\
\hline 142.0 & 61.3 & 93.5 & 67.7 \\
\hline 140.0 & 54.7 & 100.1 & 67.1 \\
\hline 138.0 & 51.6 & 106.8 & 67.7 \\
\hline 136.0 & 53.1 & 109.7 & 66.9 \\
\hline 134.0 & 52.7 & 118.5 & 65.6 \\
\hline 132.0 & 56.0 & 124.2 & 63.8 \\
\hline 130.0 & 51.8 & 126.9 & 62.4 \\
\hline 128.0 & 53.9 & 113.0 & 63.6 \\
\hline 126.0 & 56.0 & 109.2 & 65.8 \\
\hline 124.0 & 66.7 & 111.7 & 64.3 \\
\hline 122.0 & 71.7 & 124.3 & 61.0 \\
\hline 120.0 & 66.3 & 132.7 & 57.5 \\
\hline
\end{tabular}




\begin{tabular}{|c|c|c|c|}
\hline 118.0 & 77.2 & 126.4 & 56.4 \\
\hline 116.0 & 79.9 & 111.3 & 56.7 \\
\hline 114.0 & 74.1 & 87.2 & 60.1 \\
\hline 112.0 & 75.0 & 83.8 & 59.8 \\
\hline 110.0 & 71.2 & 88.5 & 60.6 \\
\hline 108.0 & 71.7 & 94.8 & 61.9 \\
\hline 106.0 & 66.3 & 101.7 & 61.1 \\
\hline 104.0 & 69.2 & 102.6 & 61.3 \\
\hline 102.0 & 59.3 & 113.4 & 61.2 \\
\hline 100.0 & 53.5 & 139.8 & 59.2 \\
\hline 98.0 & 46.7 & 155.1 & 57.9 \\
\hline 96.0 & 49.3 & 154.9 & 55.7 \\
\hline 94.0 & 44.4 & 162.5 & 54.1 \\
\hline 92.0 & 45.6 & 146.5 & 54.3 \\
\hline 90.0 & 46.9 & 150.9 & 55.0 \\
\hline 88.0 & 55.5 & 125.5 & 59.4 \\
\hline 86.0 & 56.0 & 120.7 & 59.7 \\
\hline 84.0 & 64.2 & 112.1 & 60.5 \\
\hline 82.0 & 74.1 & 107.3 & 62.8 \\
\hline 80.0 & 80.7 & 108.2 & 63.1 \\
\hline 78.0 & 89.4 & 101.8 & 64.2 \\
\hline 76.0 & 85.3 & 95.2 & 62.2 \\
\hline 74.0 & 79.9 & 96.9 & 63.5 \\
\hline 72.0 & 79.5 & 102.7 & 62.5 \\
\hline 70.0 & 77.0 & 120.0 & 58.8 \\
\hline 68.0 & 83.6 & 147.8 & 52.0 \\
\hline 66.0 & 67.5 & 172.7 & 47.9 \\
\hline 64.0 & 66.6 & 180.9 & 46.6 \\
\hline 62.0 & 65.6 & 184.2 & 45.9 \\
\hline 60.0 & 62.7 & 176.5 & 46.9 \\
\hline 58.0 & 63.2 & 157.8 & 49.8 \\
\hline 56.0 & 64.1 & 142.7 & 51.8 \\
\hline 54.0 & 56.4 & 114.4 & 56.9 \\
\hline 52.0 & 50.1 & 124.4 & 58.8 \\
\hline 50.0 & 45.3 & 137.5 & 58.9 \\
\hline 48.0 & 43.1 & 147.4 & 59.3 \\
\hline 46.0 & 42.4 & 153.4 & 58.8 \\
\hline 44.0 & 41.9 & 155.0 & 56.9 \\
\hline 42.0 & 41.4 & 156.6 & 55.4 \\
\hline 40.0 & 42.8 & 172.6 & 51.3 \\
\hline 38.0 & 48.2 & 183.5 & 46.2 \\
\hline 36.0 & 51.6 & & \\
\hline 34.0 & 46.7 & 158.3 & 52.3 \\
\hline
\end{tabular}




\begin{tabular}{|r|r|r|r|}
\hline 32.0 & 37.5 & 166.4 & 53.3 \\
\hline 30.0 & 34.6 & 168.7 & 51.0 \\
\hline 28.0 & 31.7 & 168.2 & 48.9 \\
\hline 26.0 & 33.6 & 168.2 & 48.5 \\
\hline 24.0 & 33.6 & 166.5 & 47.0 \\
\hline 22.0 & 37.0 & 163.5 & 47.3 \\
\hline 20.0 & 37.0 & 160.8 & 45.8 \\
\hline 18.0 & 51.1 & 135.6 & 49.4 \\
\hline 16.0 & 52.0 & 116.4 & 49.2 \\
\hline 14.0 & 51.1 & 116.8 & 48.1 \\
\hline 12.0 & 49.1 & 118.6 & 48.9 \\
\hline 10.0 & 69.9 & 123.8 & 59.6 \\
\hline 8.0 & 70.9 & 101.3 & 67.2 \\
\hline 6.0 & 131.9 & 75.0 & 59.3 \\
\hline 4.0 & 124.7 & 47.8 & 68.4 \\
\hline 2.0 & 151.3 & 15.5 & 75.6 \\
\hline & & & \\
\hline
\end{tabular}


Snowpit P5

\begin{tabular}{|c|c|c|c|}
\hline Depth, cm & $\mathrm{w}\left(\mathrm{NO}_{3}^{-}\right), \mathrm{ng} \mathrm{g}^{-1}$ & $\delta^{15} \mathrm{~N}, \%$ & $\delta^{18} \mathrm{O}, \%$ \\
\hline 200.0 & 20.4 & 209.6 & 73.8 \\
\hline 198.0 & 21.3 & 212.6 & 67.3 \\
\hline 196.0 & 23.0 & 223.0 & 65.1 \\
\hline 194.0 & 24.7 & 230.2 & 64.5 \\
\hline 192.0 & 25.7 & 214.2 & 59.8 \\
\hline 190.0 & 26.2 & 225.9 & 62.6 \\
\hline 188.0 & 25.7 & 221.7 & 62.3 \\
\hline 186.0 & 24.7 & 182.8 & 54.7 \\
\hline 184.0 & 22.8 & 230.5 & 67.1 \\
\hline 182.0 & 20.4 & 216.5 & 64.1 \\
\hline 180.0 & 22.3 & & \\
\hline 178.0 & 20.9 & 185.1 & 62.6 \\
\hline 176.0 & 18.4 & 198.0 & 68.1 \\
\hline 174.0 & 24.7 & 185.8 & 66.7 \\
\hline 172.0 & 21.3 & 155.9 & 55.5 \\
\hline 170.0 & 17.9 & 170.2 & 52.5 \\
\hline 168.0 & 18.9 & 178.0 & 52.7 \\
\hline 166.0 & 18.4 & 173.3 & 50.3 \\
\hline 164.0 & 20.4 & 155.1 & 47.8 \\
\hline 162.0 & 14.6 & & \\
\hline 160.0 & 18.4 & 242.5 & 71.4 \\
\hline 158.0 & 21.8 & 239.9 & 71.0 \\
\hline 156.0 & 23.3 & 202.2 & 63.5 \\
\hline 154.0 & 21.3 & 195.0 & 61.0 \\
\hline 152.0 & 22.3 & 207.7 & 58.7 \\
\hline 150.0 & 20.9 & 237.4 & 55.0 \\
\hline 148.0 & 27.2 & 239.2 & 48.1 \\
\hline 146.0 & 27.2 & 274.6 & 49.3 \\
\hline 144.0 & 27.2 & 280.4 & 48.2 \\
\hline 142.0 & 24.7 & 248.6 & 56.4 \\
\hline 140.0 & 21.8 & & \\
\hline 138.0 & 25.7 & 248.3 & 52.8 \\
\hline 136.0 & 26.2 & 213.5 & 47.1 \\
\hline 134.0 & 28.1 & 204.6 & 44.5 \\
\hline 132.0 & 33.9 & 243.4 & 49.6 \\
\hline 130.0 & 33.5 & 204.2 & 50.3 \\
\hline 128.0 & 35.4 & 232.4 & 58.9 \\
\hline 126.0 & 35.9 & 230.3 & 60.6 \\
\hline 124.0 & 33.9 & & \\
\hline 122.0 & 37.8 & 247.0 & 52.3 \\
\hline 120.0 & 38.8 & 236.9 & 51.2 \\
\hline
\end{tabular}




\begin{tabular}{|c|c|c|c|}
\hline 118.0 & 40.2 & 227.7 & 52.0 \\
\hline 116.0 & 38.8 & 210.6 & 54.8 \\
\hline 114.0 & 37.8 & 201.3 & 57.1 \\
\hline 112.0 & 36.4 & 198.3 & 59.8 \\
\hline 110.0 & 34.9 & 196.7 & 58.9 \\
\hline 108.0 & 33.5 & 190.0 & 58.0 \\
\hline 106.0 & 31.0 & 200.0 & 60.2 \\
\hline 104.0 & 28.1 & 203.2 & 59.3 \\
\hline 102.0 & 32.0 & 199.7 & 55.5 \\
\hline 100.0 & 32.5 & 227.2 & 53.7 \\
\hline 98.0 & 34.4 & 231.5 & 51.9 \\
\hline 96.0 & 35.4 & 236.8 & 50.7 \\
\hline 94.0 & 33.9 & 239.5 & 50.9 \\
\hline 92.0 & 31.0 & 242.7 & 50.1 \\
\hline 90.0 & 30.1 & 246.1 & 49.9 \\
\hline 88.0 & 30.1 & 238.9 & 50.6 \\
\hline 86.0 & 28.6 & 225.5 & 52.7 \\
\hline 84.0 & 27.6 & 228.5 & 53.1 \\
\hline 82.0 & 27.2 & 238.7 & 49.3 \\
\hline 80.0 & 29.1 & 235.6 & 47.1 \\
\hline 78.0 & 30.5 & 228.5 & 49.1 \\
\hline 76.0 & 26.2 & 235.1 & 49.8 \\
\hline 74.0 & 24.7 & 236.1 & 48.8 \\
\hline 72.0 & 26.7 & 220.8 & 50.1 \\
\hline 70.0 & 19.4 & 250.1 & 52.0 \\
\hline 68.0 & 20.4 & 237.2 & 52.0 \\
\hline 66.0 & 18.9 & 186.7 & 43.9 \\
\hline 64.0 & 22.3 & 197.4 & 43.2 \\
\hline 62.0 & 25.2 & 211.8 & 48.2 \\
\hline 60.0 & 35.9 & 221.8 & 50.1 \\
\hline 58.0 & 36.4 & 228.0 & 47.2 \\
\hline 56.0 & 50.4 & 201.4 & 46.8 \\
\hline 54.0 & 53.3 & 188.3 & 44.0 \\
\hline 52.0 & 53.8 & 190.6 & 44.2 \\
\hline 50.0 & 56.2 & 193.1 & 47.7 \\
\hline 48.0 & 65.4 & 193.0 & 50.0 \\
\hline 46.0 & 61.1 & 193.5 & 49.4 \\
\hline 44.0 & 56.7 & 192.0 & 49.1 \\
\hline 42.0 & 52.4 & 190.9 & 47.9 \\
\hline 40.0 & 50.9 & 188.5 & 46.3 \\
\hline 38.0 & 53.8 & 170.3 & 45.6 \\
\hline 36.0 & 58.7 & 162.9 & 43.7 \\
\hline 34.0 & 66.9 & 156.9 & 42.7 \\
\hline
\end{tabular}




\begin{tabular}{|r|r|r|r|}
\hline 32.0 & 67.4 & 146.7 & 45.8 \\
\hline 30.0 & 72.5 & 130.8 & 49.0 \\
\hline 28.0 & 77.6 & 123.3 & 49.8 \\
\hline 26.0 & 75.1 & 124.2 & 49.7 \\
\hline 24.0 & 69.0 & 126.1 & 50.0 \\
\hline 22.0 & 54.7 & 142.4 & 51.1 \\
\hline 20.0 & 72.5 & 158.8 & 46.9 \\
\hline 18.0 & 89.3 & 138.2 & 44.3 \\
\hline 16.0 & 111.6 & 125.5 & 45.6 \\
\hline 14.0 & 118.2 & 125.2 & 45.9 \\
\hline 12.0 & 109.6 & 127.9 & 45.2 \\
\hline 10.0 & 118.7 & 48.0 & 72.8 \\
\hline 8.0 & 144.7 & 58.8 & 82.2 \\
\hline 6.0 & 134.0 & 64.7 & 82.0 \\
\hline 4.0 & 134.0 & 65.7 & 79.1 \\
\hline 2.0 & 175.1 & 51.9 & 72.7 \\
\hline & & & \\
\hline
\end{tabular}


Snowpit P6

\begin{tabular}{|c|c|c|c|}
\hline Depth, cm & $\mathrm{w}\left(\mathrm{NO}_{3}^{-}\right), \mathrm{ng} \mathrm{g}^{-1}$ & $\delta^{15} \mathrm{~N}, \%$ & $\delta^{18} \mathrm{O}, \%$ \\
\hline 200.0 & 48.3 & 236.8 & 47.2 \\
\hline 197.5 & 39.2 & 215.3 & 51.6 \\
\hline 195.0 & 37.0 & 220.0 & 50.6 \\
\hline 192.5 & 35.4 & 226.7 & 50.9 \\
\hline 190.0 & 42.4 & 230.6 & 51.9 \\
\hline 187.5 & 43.4 & 233.8 & 52.3 \\
\hline 185.0 & 43.4 & 226.8 & 53.9 \\
\hline 182.5 & 35.9 & 220.4 & 54.3 \\
\hline 180.0 & 39.2 & 207.7 & 54.1 \\
\hline 177.5 & 37.8 & 205.1 & 53.8 \\
\hline 175.0 & 48.3 & 193.5 & 51.2 \\
\hline 172.5 & 53.1 & 187.6 & 49.6 \\
\hline 170.0 & 65.4 & 173.4 & 52.1 \\
\hline 167.5 & 70.8 & 177.2 & 53.1 \\
\hline 165.0 & 70.8 & 171.6 & 52.9 \\
\hline 162.5 & 72.9 & 165.4 & 53.0 \\
\hline 160.0 & 75.1 & 163.1 & 52.0 \\
\hline 157.5 & 70.2 & 166.3 & 51.0 \\
\hline 155.0 & 60.1 & 174.4 & 50.8 \\
\hline 152.5 & 54.2 & 194.6 & 49.0 \\
\hline 150.0 & 49.3 & 219.5 & 47.7 \\
\hline 147.5 & 54.7 & 222.0 & 43.9 \\
\hline 145.0 & 63.3 & 204.6 & 40.1 \\
\hline 142.5 & 57.4 & 188.4 & 46.0 \\
\hline 140.0 & 50.4 & 204.6 & 48.4 \\
\hline 137.5 & 49.9 & 215.6 & 47.3 \\
\hline 135.0 & 49.9 & 211.5 & 45.7 \\
\hline 132.5 & 57.4 & 196.0 & 44.3 \\
\hline 130.0 & 61.7 & 182.0 & 44.7 \\
\hline 127.5 & 60.6 & 180.9 & 44.7 \\
\hline 125.0 & 61.7 & 185.6 & 44.8 \\
\hline 122.5 & 54.2 & 187.9 & 45.0 \\
\hline 120.0 & 47.2 & 191.2 & 46.3 \\
\hline 117.5 & 49.9 & 188.9 & 47.3 \\
\hline 115.0 & 46.7 & 179.8 & 48.5 \\
\hline 112.5 & 52.0 & 179.3 & 45.5 \\
\hline 110.0 & 67.6 & 177.0 & 41.9 \\
\hline 107.5 & 76.1 & 175.9 & 39.6 \\
\hline 105.0 & 94.9 & 175.5 & 39.1 \\
\hline 102.5 & 72.4 & 149.5 & 47.0 \\
\hline 100.0 & 76.1 & 138.0 & 51.0 \\
\hline
\end{tabular}




\begin{tabular}{|c|c|c|c|}
\hline 97.5 & 68.6 & 132.3 & 50.7 \\
\hline 95.0 & 64.3 & 139.7 & 49.4 \\
\hline 92.5 & 62.2 & 157.4 & 49.5 \\
\hline 90.0 & 60.1 & 172.7 & 48.4 \\
\hline 87.5 & 63.8 & 184.8 & 46.1 \\
\hline 85.0 & 76.1 & 199.2 & 38.6 \\
\hline 82.5 & 89.5 & 192.3 & 36.2 \\
\hline 80.0 & 84.2 & 166.4 & 43.4 \\
\hline 77.5 & 96.8 & 134.1 & 46.4 \\
\hline 75.0 & 88.5 & 94.6 & 52.2 \\
\hline 72.5 & 92.2 & 98.8 & 55.3 \\
\hline 70.0 & 89.5 & 116.2 & 53.3 \\
\hline 67.5 & 90.6 & 130.7 & 54.6 \\
\hline 65.0 & 85.2 & 142.0 & 55.2 \\
\hline 62.5 & 77.2 & 144.5 & 53.7 \\
\hline 60.0 & 72.9 & 142.2 & 53.8 \\
\hline 57.5 & 86.9 & 158.3 & 47.7 \\
\hline 55.0 & 142.1 & 176.5 & 40.9 \\
\hline 52.5 & 144.5 & 182.1 & 40.0 \\
\hline 50.0 & 124.4 & 173.4 & 46.8 \\
\hline 47.5 & 115.3 & 169.3 & 57.1 \\
\hline 45.0 & 105.6 & 174.6 & 55.2 \\
\hline 42.5 & 107.2 & 153.5 & 51.0 \\
\hline 40.0 & 144.2 & 105.5 & 46.5 \\
\hline 37.5 & 159.7 & 93.8 & 46.3 \\
\hline 35.0 & 134.0 & 58.2 & 64.1 \\
\hline 32.5 & 135.1 & 57.7 & 75.7 \\
\hline 30.0 & 152.8 & 75.3 & 77.5 \\
\hline 27.5 & 154.7 & 81.6 & 77.7 \\
\hline 25.0 & 143.7 & 86.4 & 74.4 \\
\hline 22.5 & 125.4 & 89.7 & 72.0 \\
\hline 20.0 & 117.4 & 100.0 & 68.4 \\
\hline 17.5 & 108.3 & 110.7 & 66.2 \\
\hline 15.0 & 104.5 & 115.8 & 65.7 \\
\hline 12.5 & 95.4 & 121.6 & 64.2 \\
\hline \multicolumn{4}{|l|}{10.0} \\
\hline 7.5 & 196.2 & 76.2 & 81.9 \\
\hline 5.0 & 168.9 & 90.0 & 80.2 \\
\hline 2.5 & 202.6 & 76.8 & 70.5 \\
\hline
\end{tabular}


Snowpit P7

\begin{tabular}{|c|c|c|c|}
\hline Depth, cm & $\mathrm{w}\left(\mathrm{NO}_{3}^{-}\right), \mathrm{ng} \mathrm{g}^{-1}$ & $\delta^{15} \mathrm{~N}, \%$ & $\delta^{18} \mathrm{O}, \%$ \\
\hline 300.0 & 15.6 & 312.6 & 64.6 \\
\hline 297.5 & 16.1 & 329.7 & 59.5 \\
\hline 295.0 & 16.5 & 334.5 & 61.2 \\
\hline 292.5 & 16.1 & 338.2 & 61.2 \\
\hline 290.0 & 15.1 & 341.3 & 60.5 \\
\hline 287.5 & 16.4 & 331.5 & 61.0 \\
\hline 285.0 & 15.5 & 318.9 & 64.1 \\
\hline 282.5 & 14.0 & 301.6 & 65.6 \\
\hline 280.0 & 12.9 & 309.1 & 67.1 \\
\hline 277.5 & 13.4 & 297.5 & 63.4 \\
\hline 275.0 & 13.9 & 305.8 & 66.8 \\
\hline 272.5 & 13.3 & 296.0 & 66.1 \\
\hline 270.0 & 13.0 & 312.5 & 64.0 \\
\hline 267.5 & 13.4 & 315.2 & 64.5 \\
\hline 265.0 & 13.8 & 319.0 & 63.5 \\
\hline 262.5 & 15.4 & 328.1 & 61.0 \\
\hline 260.0 & 13.9 & 322.1 & 54.5 \\
\hline 257.5 & 15.3 & 326.9 & 53.1 \\
\hline 255.0 & 15.5 & 332.6 & 53.8 \\
\hline 252.5 & 15.5 & 338.6 & 52.3 \\
\hline 250.0 & 15.5 & 352.6 & 51.5 \\
\hline 247.5 & 14.8 & 354.8 & 51.4 \\
\hline 245.0 & 14.0 & 347.0 & 54.1 \\
\hline 242.5 & 13.1 & 328.7 & 56.2 \\
\hline 240.0 & 12.4 & 313.9 & 56.5 \\
\hline 237.5 & 11.3 & 316.1 & 59.1 \\
\hline 235.0 & 11.6 & 306.9 & 59.4 \\
\hline 232.5 & 11.2 & 295.2 & 59.1 \\
\hline 230.0 & 13.0 & 329.3 & 57.2 \\
\hline 227.5 & 13.7 & 333.7 & 54.6 \\
\hline 225.0 & 13.5 & 361.1 & 49.7 \\
\hline 222.5 & 13.4 & 361.1 & 49.1 \\
\hline 220.0 & 13.5 & 348.5 & 50.1 \\
\hline 217.5 & 12.8 & 333.5 & 52.7 \\
\hline 215.0 & 11.6 & 328.4 & 54.6 \\
\hline 212.5 & 12.4 & 318.0 & 56.3 \\
\hline 210.0 & 11.1 & 308.3 & 56.9 \\
\hline 207.5 & 11.2 & 296.7 & 57.6 \\
\hline 205.0 & 10.6 & 309.8 & 55.7 \\
\hline 202.5 & 11.9 & 322.8 & 53.7 \\
\hline 200.0 & 13.3 & 344.9 & 51.0 \\
\hline
\end{tabular}




\begin{tabular}{|c|c|c|c|}
\hline 197.5 & 12.5 & 368.2 & 57.4 \\
\hline 195.0 & 12.6 & 341.2 & 52.2 \\
\hline 192.5 & 10.9 & 334.5 & 55.8 \\
\hline 190.0 & 14.0 & 311.0 & 55.3 \\
\hline 187.5 & 10.4 & 307.6 & 55.4 \\
\hline 185.0 & 11.1 & 300.4 & 57.9 \\
\hline 182.5 & 9.6 & 297.9 & 61.0 \\
\hline 180.0 & 9.9 & 299.6 & 61.2 \\
\hline 177.5 & 9.1 & 283.2 & 61.3 \\
\hline 175.0 & 9.4 & 283.8 & 59.8 \\
\hline 172.5 & 10.4 & 291.9 & 57.1 \\
\hline 170.0 & 10.1 & 305.4 & 56.2 \\
\hline 167.5 & 12.6 & 311.2 & 53.9 \\
\hline 165.0 & 10.3 & 315.8 & 51.4 \\
\hline 162.5 & 12.6 & 343.0 & 47.9 \\
\hline 160.0 & 14.9 & 337.4 & 47.8 \\
\hline 157.5 & 11.6 & 393.8 & 45.7 \\
\hline 155.0 & 12.3 & 370.9 & 47.3 \\
\hline 152.5 & 14.0 & 342.8 & 49.5 \\
\hline 150.0 & 13.2 & 332.9 & 50.4 \\
\hline 147.5 & 12.0 & 397.4 & 44.0 \\
\hline 145.0 & 14.9 & 402.6 & 42.5 \\
\hline 142.5 & 12.6 & 397.3 & 44.4 \\
\hline 140.0 & 10.7 & 365.0 & 47.2 \\
\hline 137.5 & 11.6 & 353.2 & 50.1 \\
\hline 135.0 & 11.3 & 370.8 & 50.1 \\
\hline 132.5 & 10.6 & 354.2 & 52.2 \\
\hline 130.0 & 11.7 & 325.5 & 57.1 \\
\hline 127.5 & 12.7 & 331.8 & 53.8 \\
\hline 125.0 & 11.2 & 324.2 & 51.3 \\
\hline 122.5 & 10.8 & 329.3 & 50.7 \\
\hline 120.0 & 12.3 & 351.1 & 47.3 \\
\hline 117.5 & 12.0 & 343.6 & 46.1 \\
\hline 115.0 & 13.5 & 333.8 & 45.7 \\
\hline 112.5 & 9.1 & 344.8 & 45.6 \\
\hline 110.0 & 10.2 & 347.1 & 43.3 \\
\hline 107.5 & 10.8 & 352.2 & 41.4 \\
\hline 105.0 & 9.9 & 361.6 & 42.1 \\
\hline 102.5 & 9.6 & 366.9 & 42.5 \\
\hline 100.0 & 8.9 & 339.4 & 43.6 \\
\hline 97.5 & 7.9 & 332.4 & 43.7 \\
\hline 95.0 & 8.1 & 322.8 & 44.0 \\
\hline 92.5 & 7.9 & 337.2 & 40.9 \\
\hline
\end{tabular}




\begin{tabular}{|c|c|c|c|}
\hline 90.0 & 9.6 & 338.7 & 39.8 \\
\hline 87.5 & 9.5 & 335.6 & 41.0 \\
\hline 85.0 & 14.0 & 398.5 & 30.1 \\
\hline 82.5 & 13.4 & 396.5 & 31.3 \\
\hline 80.0 & 13.1 & 366.0 & 34.0 \\
\hline 77.5 & 14.6 & 361.5 & 36.4 \\
\hline 75.0 & 15.4 & 394.9 & 35.5 \\
\hline 72.5 & 18.3 & 394.2 & 31.2 \\
\hline 70.0 & 17.9 & 370.6 & 34.7 \\
\hline 67.5 & 17.7 & 388.8 & 37.3 \\
\hline 65.0 & 19.4 & 374.0 & 36.0 \\
\hline 62.5 & 20.5 & 362.9 & 35.9 \\
\hline 60.0 & 19.8 & 367.3 & 35.8 \\
\hline 57.5 & 27.4 & 374.5 & 35.7 \\
\hline 55.0 & 30.4 & 436.5 & 35.4 \\
\hline 52.5 & 29.2 & 460.8 & 33.3 \\
\hline 50.0 & 23.1 & 453.0 & 30.5 \\
\hline 47.5 & 21.5 & 420.9 & 30.2 \\
\hline 45.0 & 22.7 & 430.9 & 21.4 \\
\hline 42.5 & 27.4 & 437.0 & 16.8 \\
\hline 40.0 & 23.7 & 436.2 & 18.1 \\
\hline 37.5 & 22.6 & 402.2 & 23.6 \\
\hline 35.0 & 20.6 & 388.6 & 27.5 \\
\hline 32.5 & 19.0 & 355.4 & 34.7 \\
\hline 30.0 & 18.4 & 347.9 & 34.9 \\
\hline 27.5 & 39.6 & 282.0 & 38.4 \\
\hline 25.0 & 42.3 & 265.1 & 40.4 \\
\hline 22.5 & 37.6 & 259.5 & 42.6 \\
\hline 20.0 & 32.5 & 272.3 & 41.2 \\
\hline 17.5 & 65.8 & 269.6 & 32.6 \\
\hline 15.0 & 66.5 & 293.6 & 33.2 \\
\hline 12.5 & 67.0 & 287.8 & 30.5 \\
\hline 10.0 & 123.3 & 189.1 & 63.0 \\
\hline 7.5 & 158.9 & 185.6 & 57.6 \\
\hline 5.0 & 319.2 & 129.8 & 84.0 \\
\hline 2.5 & 373.8 & 111.0 & 74.9 \\
\hline
\end{tabular}




\section{References}

Ding, M., Xiao, C., Jin, B., Ren, J., Qin, D., and Sun, W.: Distribution of $\delta^{18} \mathrm{O}$ in surface snow along a transect from Zhongshan Station to Dome A, East Antarctica, Chin. Sci. Bull., 55, 2709-2714, 2010.

Ding, M., Xiao, C., Li, Y., Ren, J., Hou, S., Jin, B., and Sun, B.: Spatial variability of surface mass balance along a traverse route from Zhongshan station to Dome A, Antarctica, J. Glaciol., 57, 658-666, 2011.

Erbland, J., Vicars, W., Savarino, J., Morin, S., Frey, M., Frosini, D., Vince, E., and Martins, J.: Air-snow transfer of nitrate on the East Antarctic Plateau - Part 1: Isotopic evidence for a photolytically driven dynamic equilibrium in summer, Atmos. Chem. Phys., 13, 6403-6419, 2013.

Frey, M. M., Savarino, J., Morin, S., Erbland, J., and Martins, J.: Photolysis imprint in the nitrate stable isotope signal in snow and atmosphere of East Antarctica and implications for reactive nitrogen cycling, Atmos. Chem. Phys., 9, 8681-8696, 2009. 